Proc. 13th Econophysics Colloquium (EC) and 9th Symposium of Physics in Economy and Social Sciences (FENS), 2017

\title{
Structural Transformation of Minimal Spanning Trees in World Commodity Market
}

\author{
J.W. $\mathrm{LEE}^{a, *}$ AND A. NOBI ${ }^{b}$ \\ ${ }^{a}$ Department of Physics, Inha University, Incheon, Korea \\ ${ }^{b}$ Department of Computer Science and Telecommunication Engineering, \\ Noakhali Science and Technology University, Sonapur, Bangladesh
}

\begin{abstract}
We consider structural change of a world trade-flow network for different commodities from 1995 to 2013 . A minimum spanning tree is generated from the trade flow data, and we investigate the hierarchical organization of the tree by changing the hub node. Only a few countries are the hub nodes for many of the commodities. We observed the structural transitions, from a chain-like tree (many countries are hub nodes) to a bi-starlike tree (only two countries are hub nodes). In the bi-starlike tree, China and Germany are the only two hub nodes.
\end{abstract}

DOI: 10.12693 /APhysPolA.133.1414

PACS/topics: 89.20.-a, 89.65.Gh, 89.75.-k

\section{Introduction}

The concept of complex networks has been applied to socio-technical networks [1-4], the world-trade network [5, 6], world commodity markets [7, 8], and stock markets [9-11]. The economic activities of countries are projected onto the world trade in commodities. Cha et al. observed the disparity and patterns of world-trade networks [5]. The redistribution of wealth was observed in backbone networks of world trade [6]. Industrialized countries like the USA, Germany, Japan, and those in Europe trade many commodities with other countries in the world. Nobi et al. reported the dynamics of the consumer group and the response of commodity markets by using principal component analysis [7]. Nobi and Lee observed the financial states of world financial and commodities markets around the sovereign debt crisis [8]. Systemic risk and the transitions of financial networks were reported for complex networks of the stock market [9-11].

Trading networks are heavily connected, complex networks. Therefore, it is hard to see the relations between countries and the positions of each country in the networks. The properties of a scale-free network were observed in the world trade web (WTW) or international trade networks (ITNs) [12-19]. Serrano and Boguna reported that the world trade web displays scalefree and small-world properties [12]. Li et al. observed scale-free degree distribution and small-world properties in the world trade web [13]. Duan introduced a modified betweenness centrality to measure the trade flow in world-trade networks [14]. Garlaschelli and Loffredo reported that the power law distribution of the gross domestic product can be identified with fitness determining the topology of the WTW $[15,16]$. Squartini

${ }^{*}$ corresponding author; e-mail: jaewlee@inha.ac.kr et al. showed that the degree sequence is the most informative of the binary properties in ITNs [17]. Kantar et al. reported on the hierarchical structure of Turkey's foreign trade and on clustering of countries in trade [18]. Shutters and Muneepeerakul observed two distinct classes in the agricultural-trade network based on triadic frequencies [19].

The structural properties of world commodity networks were observed for all world commodities [2023]. Higalgo et al. observed the network relations between products in world commodity markets [20]. They found that the more influential products are located in the core of the network. The heterogeneity and community structure of complex networks were reported for commodity-specific international trade [21, 22]. The trade network for crude oil showed small-world and flat-world properties [23].

In this work, we extracted a minimal spanning tree (MST) from a time series of the world commodity market. We observed changes in the hub node of the MST for the commodity market. This article is organized as follows. In Sect. 2, we introduce the data set we analyzed. In Sect. 3, we give the analyzed results of the MST. We offer concluding remarks in Sect. 4.

\section{Data set}

We analyzed international trade data provided by the United Nations Commodity Trade Statistics Database (UN Comtrade) [24]. The data for eight types of commodities according to Standard International Trade Classification Rev 2 (SITC 2) were analyzed for $N=170$ countries from 1995 to 2013. The commodities are classified as follows: (1) food and live animals chiefly for food, (2) beverages and tobacco, (3) crude materials (inedible), except fuels, (4) mineral fuels, lubricants, and related materials, (5) animal and vegetable oils, fats, and waxes, (6) chemicals and related products, (7) manufactured goods chiefly classified by material, and (8) machinery 
and transport equipment. We also analyzed trade data for all commodities. In the Comtrade data set, the quantities of imports and exports between two countries are recorded for each commodity in each year. The trading values of commodities between two countries are represented by the interacting strength, $m_{i j}(t)$, in a year, $t$, where one country, $i$, exports a commodity to another country, $j$. Then, $m_{j i}(t)$ is the quantity of imports from $j$ to $i$. Therefore, we define the link strength between two nodes by the average of the exports and imports between the two countries. We extracted a minimal spanning tree and observed a change of the hub node in the market. By using the link strength, we applied Kruskal's algorithm to extract the minimal spanning tree [25]. The MST is a tree structure with $N-1$ links without loops in the network. In the MST, the links are ordered according to the highest weights. It is possible to generate some networks by considering exports only or imports only. In this work, we focus on total trade between two countries.

\section{Results}

The minimal spanning tree was generated from a data set of international trading quantities. We extracted the minimal spanning tree by using Kruskal's algorithm [25]. From the MST, we can observe the change in the world trading properties from 1995 to 2013. The MST has a total number of nodes, $N=170$, and links, $L=169$, which are the most dominant trading links among the countries.

In Fig. 1, we represent the MST in the year 1995 for manufactured goods classified chiefly by material. The MST has the structure of a long, connected chain with local hubs. We define the hub node as the node with the highest degree in the MST. For example, node number 59 in Fig. 1 is Germany, which is a super-hub in the MST. The second hub is node number 80 (Japan). China (node number 33) is located outside the boundary for the year 1993. From the structure of the MST, we expect to see the local trading block around the local hub. Therefore, the MST has an elongated tree structure.

The structure of the MST in 2013 changed to a bistarlike structure with two super-hubs in the center of the tree, as shown in Fig. 2. The super-hub node is China (number 33) in the MST. The main trading links of the countries for manufactured goods classified chiefly by material are connected to China directly or indirectly. The second hub is Germany (number 59) connecting European countries in the MST. When we monitored the hub node in the MST, we observed a change in status in the MST for all commodities from Germany to China in 2004. Before 2004, Germany is the hub node, but not a super-hub. There are many hubs, such as France, Japan, South Korea, and the USA. China is located on the periphery of the MSTs. However, since 2004, China has become a super-hub in the MSTs for all commodities, as shown in Fig. 2. Many countries are connected to China. This indicates that China takes the position of a world factory.

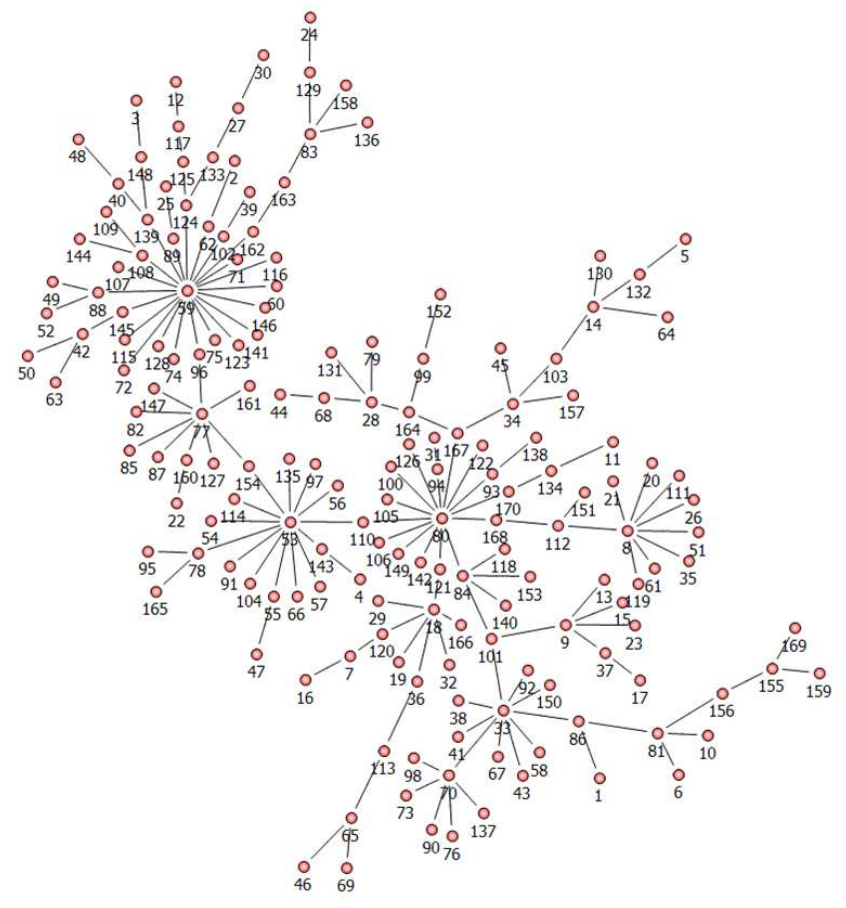

Fig. 1. Typical structures of the minimal spanning tree observed in the trading networks. A chain-like tree is seen for 1995 of manufactured goods classified chiefly by material, where the hub is node Germany (59) at the boundary of the tree, and node number 80 is Japan.

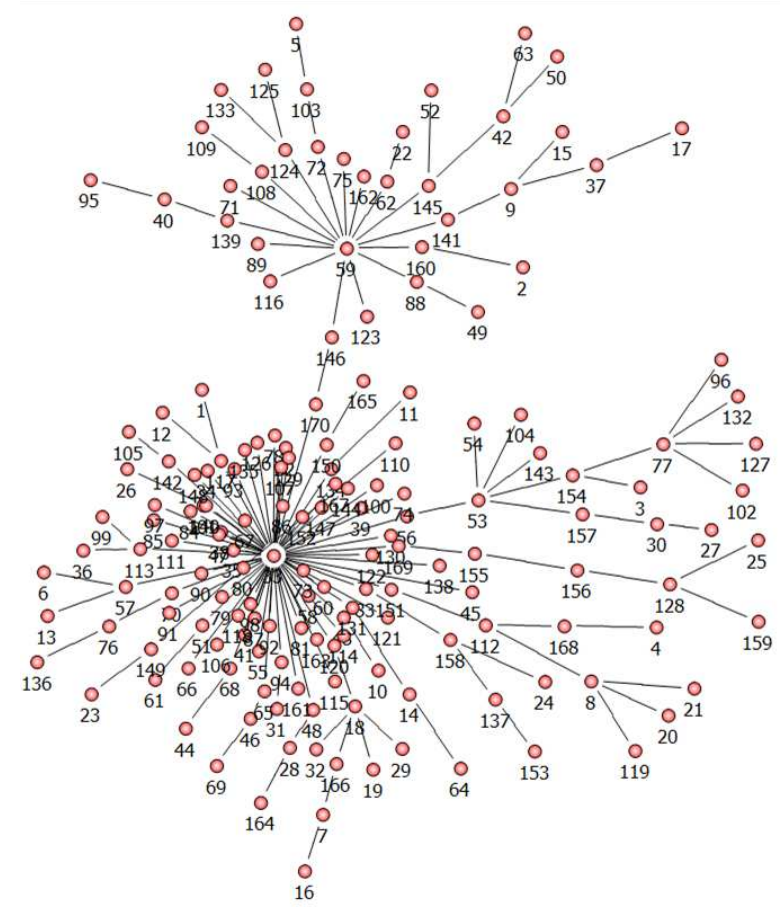

Fig. 2. Change in the minimal spanning tree is observed for trading networks. The bi-starlike tree is seen for manufactured goods classified chiefly by material in 2013, where the hub is China (node number 33) and the second hub is Germany (59). 
The hub nodes in sectors of commodities are shown in Fig. 3. Different countries occupy the hub node in each category of commodities. In manufactured goods, the hub node changed from Germany to China in the year 2000. For many commodities, the structure of the networks changed heavily when China took its position as a world factory. In beverages and tobacco, France is the hub node in all analyzed years except 2012. In the chemical sector, the hub node changed from France and Germany to China in the year 2006. In machinery equipment, before 2006, Germany and Japan were hub nodes. However, after 2006, China became the hub node in machinery equipment. In mineral fuels, China has taken the hub node position since 2006. Before 2006, Australia and France were hub nodes. Only a few countries are the hub node in the MST of the world commodity market.

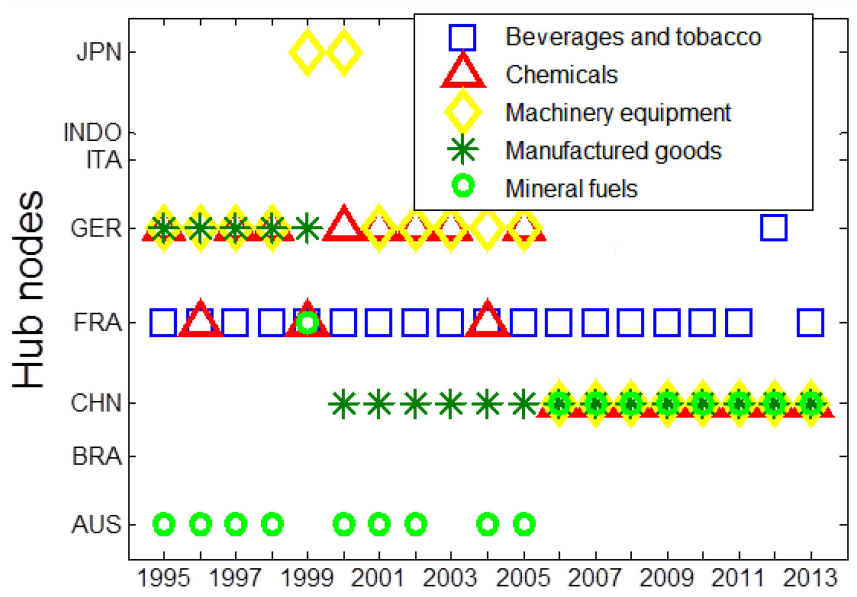

Fig. 3. The hub nodes for commodity sectors are presented over time. Different countries occupy the hub node in each category of the commodities. China takes the position of super-hub in the MST.

\section{Conclusions}

We consider a minimal spanning tree extracted from trading values of world commodities for all commodities and eight classified commodities. The trading networks are heavily connected, complex networks. We observed transitions in the trading networks. We observed structural transitions of the MST from a chain-like tree to a bi-starlike tree. In the bi-starlike tree, China and Germany are the only two hub nodes. In the industrial sectors of world trade, China took the hub node position in the trading networks for almost all commodities except beverages and tobacco around the year 2000. If we monitor the MST of world trade networks, we can identify the most influential connections between two countries and the changes in trading patterns. In this work, we considered total trade between two countries. In future work, we will consider export or import networks.

\section{Acknowledgments}

This research was supported by the Basic Science Research Program through the National Research Foundation of Korea (NRF), funded by the Ministry of Science, ICT and Future Planning (NRF-2017R1A2B4005214).

\section{References}

[1] R. Albet, A.-L. Barabasi, Rev. Mod. Phys. 74, 47 (2002).

[2] S.N. Dorogovtsev, J.F.F. Mendes, Adv. Phys. 51, 1079 (2002).

[3] S. Boccaletti, V. Latora, Y. Moreno, M. Chavez, D.U. Hwang, Phys. Rep. 424, 175 (2006).

[4] S. Boccaletti, G. Bianconi, R. Criado, C.I. del Genio, J. Gomez-Gardenes, M. Romance, I. Sendina-Nadal, Z. Wang, M. Zanin, Phys. Rep. 544, 1 (2014).

[5] M.-Y. Cha, J.W. Lee, D.-S. Lee, J. Korean Phys. Soc. 56, 998 (2010).

[6] M.-Y. Cha, J.W. Lee, D.-S. Lee, D.H. Kim, Comp. Phys. Commun. 182, 216 (2011).

[7] A. Nobi, S. Alam, J.W. Lee, Physica A 482, 337 (2017).

[8] E.U. Patwary, J.Y. Lee, A. Nobi, D.H. Kim, J.W. Lee, J. Korean Phys. Soc. 71, 444 (2017).

[9] A. Nobi, J.W. Lee, Chaos 27, 063107 (2017).

[10] A. Nobi, J.W. Lee, J. Korean Phys. Soc. 71, 733 (2017).

[11] J.W. Lee, A. Nobi, Comput. Econ. 51, 195 (2018).

[12] M.A. Serrano, M. Boguna, Phys. Rev. E 68, 015101 (2003).

[13] X. Li, Y.Y. Jin, G. Chen, Physica A 328, 287 (2003).

[14] W.-Q. Duan, Physica A 387, 519 (2008).

[15] D. Garlaschelli, M.I. Loffredo, Phys. Rev. Lett. 93, 188701 (2004).

[16] D. Garlaschelli, M.I. Loffredo, Physica A 355, 138 (2005).

[17] T. Squartini, G. Fagiolo, D. Garlaschelli, Phys. Rev. E 84, 046117 (2011).

[18] E. Kantar, B. Deviren, M. Kestin, Physica A 390 , 3454 (2011)

[19] S.T. Shutters, R. Muneepeerakul, Plos One 7, e39756 (2012).

[20] C.A. Hidalgo, B. Klinger, A.-L. Barabasi, R. Hausmann, Science 317, 482 (2007).

[21] M. Barigozzi, G. Fagiolo, D. Garlaschelli, Phys. Rev. E 81, 046104 (2010).

[22] M. Barigozzi, G. Fagiolo, G. Mangioni, Physica A 390, 2051 (2011).

[23] Y. Yang, J.P.H. Poon, Y. Liu, S. Bagchi-Sen, Energy 93, 534 (2015).

[24] UN Comtrade Database.

[25] G. Fagiolo, J. Econ. Interact. Coord. 5, 1 (2010). 